\title{
A technical review of no-reference image quality assessment algorithms for
}

\section{contrast distorted images}

DOI:10.36909/jer.11885

Preeti Mittal ${ }^{*}$, Rajesh Kumar Saini ${ }^{*}$, Justin Varghese ${ }^{* *}$, Neeraj Kumar Jain ${ }^{*}$

*Department of Mathematical Sciences and Computer Application, Bundelkhand University, Jhansi, India.

${ }^{* *}$ Karpagam College of Engineering, Coimbatore, India

Corresponding Author: preetimittal1980@yahoo.co.in

\begin{abstract}
Automatic image quality assessment similar to human vision perception is an essential process for real-time image processing applications to perform perceptual image assessments for effectively achieving their goals. As no-reference image quality assessment (NR-IQA) schemes perform perceptual assessments of images without any information about their original version, these algorithms suit real-time computer vision techniques because of the non-availability of reference images. Contrast and colorfulness play important roles in determining the quality of color images. By combining many IQA metrics, a number of combined metrics had been devised. This study provides an insight into major NR-IQA methods and their effectiveness in assessing contrast, colorfulness, and overall quality of contrast-degraded images with technical analysis. The effectiveness of top-ranking NR-IQA methods is experimentally assessed with benchmark assessment methods on images from benchmarked databases. The study provides insight into open research challenges in the area of NR-IQA for developing new promising methods by clearly demarcating the difficulties of top-ranking NR-IQA methods.
\end{abstract}

Keywords: IQA, NR-IQA, Features Extraction, Image Distortion, Machine Learning, Quality Prediction 


\section{INTRODUCTION}

Digital image processing plays a vital role in the key vision-based applications including robotics, medical imaging, agricultural development, industrial automation, surveillance and military, autonomous vehicles, tactile feedback, etc. (Gonzalez \& Woods, 2006; Mittal et al., 2019). Image quality in computer vision denotes the relative correctness of images in terms of perceptual assessments by which the imaging systems acquire, digitize, archive, compress, transmit and display images (Mohammadi et al., 2014; Gonzalez \& Woods, 2006). Automatic Image Quality Assessment (IQA) similar to the biological human vision analysis is an essential process for image processing systems to perform perceptual assessments for effectively performing its subsequent tasks such as segmentation, representation, classification, recognition, and other decision-making processes (Navas \& Sasikumar, 2011). The image quality assessment schemes perform perceptual assessments of the image in terms of its contrast, luminance, sharpness, dynamic range, color, chromatic deviations, vignetting, tone reproduction, and noise in the similar way humans perform the subjective evaluation.

In reference to the accessibility of the reference image, the three ways to perform objective image quality assessment are Full Reference Image Quality Assessment (FR-IQA), Reduced Reference Image Quality Assessment (RR-IQA), and No Reference Image Quality Assessment (NR-IQA). FR-IQA algorithms decide the quality of the input image by comparing the input image with the reference images while RR-IQA algorithms use some limited characteristics of the original image (Ma et al., 2017; Ding et al., 2021). NR-IQA schemes determine the image quality without using any prior knowledge about pristine images. In most of the real-world applications, there is the absence of reference image to compare the quality of its degraded images and hence NR-IQA metrics have prime importance in the case of real-time image processing applications. Some of the NR-IQAs can be used for general-purpose and some are designed for specific degradations. This study 
evaluates the performances of top-ranking NR-IQA schemes in assessing the contrast, colorfulness, and overall quality of the distorted color images in a general perspective.

Many NR-IQA schemes focused only on specific characteristics of image quality like entropy (Krotkov \& Martin, 1986; Xie \& Wang, 2010), brightness (Bezryadin et al., 2007), colorfulness (Yendrikhovski et al., 1998; Fu \& Shih, 2006; Hasler \& Suesstrunk, 2003; Panetta et al., 2013), sharpness (Fu \& Shih, 2006; Santos et al., 1997; Eskicioglu \& Fisher, 1995; Pech-Pacheco et al., 2000) and contrast (Xie \& Wang, 2010; Panetta et al., 2013; Santos et al., 1997; Firestone et al., 1991; Agaian et al., 2000; Wharton et al., 2006; Agaian et al., 2007; Panetta et al., 2008; Panetta et al., 2011; Wu et al., 2013). As the first NR-IQA, Fu and Shih (2006) formulated a generic Quality Index (QI) for color images by using colorfulness, sharpness, and contrast metrics. To combine these metrics, the calibration procedure is employed on subjective data. In the same direction, Panetta et al. (2013) presented Color Quality Enhancement (CQE) measure and Gao et al. (2014) suggested Color Quality Measure (CQM). Later, Xie and Wang (2010) introduced Color image Assessment Factor (CAF) by using a multiplicative model to present the quality model based on contrast, entropy, and Normalized Neighbourhood Function (NNF). With the advancement in machine learning techniques, many NR-IQA metrics were being introduced in the literature by using various variations of Support Vector Machine (SVM) (Mittal et al., 2011; Yan et al., 2019; Fang et al., 2014; Chen et al., 2019), Artificial Neural Network (ANN) (Bouzerdoum et al., 2004; Li et al., 2011; Talebi \& Milanfar, 2018) and Convolution Neural Networks (CNN) (Bosse et al., 2016; Gu et al., 2017; Liu et al., 2017).

These metrics are classified based on the awareness of distortion types and subjective opinion of distorted images at the time of training. The Distortion-Aware (DA) models are modelled by performing training on specific distortions (Mittal et al., 2012). Opinion-Aware metrics use images labelled by subjective quality scores for training. Since the majority of NR 
image quality schemes were designed to measure specific distortions for specific applications, these methods may produce unsatisfactory performance in the case of other distortions/applications.

Most of the general-purpose IQA schemes developed in the literature were tested on compression, noise, and blurring based distortions. It is seen that very few algorithms consider contrast distortion. George and Prabavathy (2014) reviewed six NR algorithms while Manap and Shao (2015) and Krishnendu et al. (2021) surveyed a small number of NR algorithms and considered the images with many types of distortions but failed to consider contrast distortions. Kamble and Bhurchandi (2015) surveyed a large number of metrics for different types of distortions but did not assess the performance of IQA algorithms on images with contrast distortion. Xu et al. (2017) reviewed the development factors of eight NR-IQA algorithms and compared their performance on three databases with limited image distortions. Ahmed et al. (2017) reviewed and compared only two algorithms suitable for assessing contrast distorted images in different transform domains. Zhai and Min (2020) studied numerous IQA algorithms on many databases but they also ignored the metrics designed specifically for contrast distortion. Varga (2021) conducted the study on numerous NR-IQA methods but only on LIVE In the Wild and KonIQ-10k databases. Wang (2021) summarizes deep learning-based NRIQA approaches but didn't review approaches designed for contrast distortion. Unlike these studies on NR-IQA, this study is unique in reviewing and experimentally comparing a large number of metrics based on different features of the images under numerous contrast-distorted and illuminating conditions. All these reviews and comparisons are made by considering the whole database as one entity while the images may be distorted with different functions. 
Artificially generated databases such as CSIQ, TID2013, and CCID2014 consist of images distorted with certain levels of specific distortions and hence the IQA schemes need to rank images with respect to the affecting distortion levels. Further, for ranking IQA schemes with respect to their performance, additional benchmarking parameters are required. Hence, these technical reviews in the literature though assess some specific goals of IQA measures but did not adequately analyse all goals of IQA methods such as effective testing on larger sets of images from numerous databases, contrast/colorfulness assessments in different illumination situations, and benchmarked ranking of IQA schemes.

By focussing on the limitations of existing technical reviews in the literature, this paper makes an extensive discussion and experimental analysis of top-ranking NR-IQA schemes in the literature for assessing the contrast, colorfulness, and overall quality of images. Unlike other studies in the literature, the paper extends its study on comparing and evaluating the performances of numerous IQA algorithms on numerous contrast distorted images. As NRIQAs are used by the researchers to quantify the image quality in the image enhancement methods, different image enhancement techniques used many metrics to assess the colorfulness and contrast of enhanced images. This study is specifically carried out on analyzing popular and effective general-purpose no-reference metrics used to quantify the colorfulness and contrast of the enhanced images. The study analyses the effectiveness of using different IQA algorithms in the case of different distortions under various imaging conditions. Further, the study uses Average Spearman Rank Order Correlation Coefficient (ASROCC) and Average Kendall Rank Order Correlation Coefficient (AKROCC) as the benchmark parameters to assess the performance of the existing NR-IQAs.

The paper is organized into six sections. Prominent IQA methods in the literature are presented in Section 2. Section 3 presents the image databases used in this study, the details of experiments, and the evaluation criteria of different algorithms. Section 4 demarcates the 
results of this study. The conclusions are presented in Section 5. Finally, Section 6 provides the future scope of the research.

\section{PROMinent NR-IQA SCHEMES}

The NR-IQA algorithms attempt to provide computational methods for performing quality assessments of images with limited/no-priori information about illumination, image background, objects, textures, features, and possible distortions. The section briefly discusses the contrast, colorfulness, and combined metrics.

\section{Colorfulness Metrics}

Colorfulness refers to chromaticity and it is defined as the visual sensation of chromatic strength in accordance with the perceived color. The colorfulness of the image is proportional to luminance, except for bright images (Palus, 2006) and it denotes the strength of the hue component of the image. This subsection details various colorfulness-based IQA methods used in the literature.

Yendrikhovski et al. (1998) proposed Colorfulness Index (CI) as the sum of the mean and standard deviation of saturation of the image in CIE LUV color space and found that the CI and human judgement have a correlation of 0.91 .

Hasler and Suesstrunk (2003) proposed the Colorfulness Index in the opponent color spaces rg \& yb and found its correlation to 95.3\%. Later, Fu and Shih (2006) normalized this colorfulness metric as

$$
\text { QI_Colorfulness }=\left(0.3 * \mu_{\mathrm{rg}, \mathrm{yb}}+\sigma_{\mathrm{rg}, \mathrm{yb}}\right) / 85.59
$$

Where, $\mu_{\mathrm{rg}, \mathrm{yb}}=\sqrt{\mu_{\mathrm{rg}}^{2}+\mu_{\mathrm{yb}}^{2}}$ and $\sigma_{\mathrm{rg}, \mathrm{yb}}=\sqrt{\sigma_{\mathrm{rg}}^{2}+\sigma_{\mathrm{yb}}^{2}}$; and $\mu_{\mathrm{rg}}, \mu_{\mathrm{yb}}, \underset{\sigma \mathrm{rg}}{2}, \sigma_{\mathrm{yb}}^{2}$ are the average and standard deviation of $\mathrm{rg}$ and yb channels.

The concept of colorfulness was further explored by Panetta et al. (2013) and two extensions of colorfulness came into existence as 


$$
\begin{aligned}
& \text { CQE_Colorfulness }{ }_{1}=0.2 \log \frac{\sigma_{\mathrm{rg}}^{2}}{\left|\mu_{\mathrm{rg}}\right|^{0.2}} \log \frac{\sigma_{\mathrm{yb}}^{2}}{\left|\mu_{\mathrm{yb}}\right|^{0.2}} \\
& \text { CQE_Colorfulness }_{2}=0.2 \log \frac{\sigma_{\mathrm{rg}}^{2} \times \sigma_{\mathrm{yb}}^{2}}{\log \sigma_{\mathrm{c}}^{2}} \log \frac{\mu_{\mathrm{rg}}^{2} \times \mu_{\mathrm{yb}}^{2}}{\log \mu_{\mathrm{c}}^{2}}
\end{aligned}
$$

where, $\mu_{\mathrm{c}}=0.5^{*}\left(\mu_{\mathrm{rg}}+\mu_{\mathrm{yb}}\right)$ and $\sigma_{\mathrm{c}}=\frac{1}{2^{*} \mathrm{M}^{* \mathrm{~N}}} \sum_{\mathrm{i}=1}^{\mathrm{M}} \sum_{\mathrm{j}=1}^{\mathrm{N}}\left[\left(\mathrm{I}_{\mathrm{rg}}^{2}(\mathrm{i}, \mathrm{j})-\mu_{\mathrm{c}}^{2}\right)+\left(\mathrm{I}_{\mathrm{yb}}^{2}(\mathrm{i}, \mathrm{j})-\mu_{\mathrm{c}}^{2}\right)\right]$

Gu et al. (2017) used Mean Saturation (MS) as a colorfulness measure. The given image for assessment is transformed into HSV color space from RGB color space and the mean of saturation channel is calculated.

\section{Contrast Metrics}

In visual perception, contrast plays a vital role in distinguishing objects and is evaluated by finding the dissimilarity in luminance of the object of interest from its background. Human perception is more responsive to contrast than luminance. Although many IQA methods identify contrast as the measure of luminance/intensity channels, very few metrics such as Average Color Contrast (ACC) and Color Root Mean Enhancement (CRME) measure contrast using color channels. Table 1 briefly describes the contrast metrics used in the study and the details can be found in their respective papers.

Table 1. Contrast metrics used in the study

\begin{tabular}{|l|c|}
\hline Contrast Metric & Definition \\
\hline Range (Firestone et al., 1991) & $\frac{1}{\mathrm{MN}} \sum \sum(\mathrm{G}(\mathrm{i}, \mathrm{j})-\overline{\mathrm{G}})^{2}$ \\
\hline $\begin{array}{l}\text { Variance (Krotkov \& Martin, } \\
\text { 1986) }\end{array}$ & $\frac{1}{\mathrm{MN}-1} \sum \sum(\mathrm{G}(\mathrm{i}, \mathrm{j})-\overline{\mathrm{G}})^{2}$ \\
\hline RMS Contrast (Peli, 1990) & Variance/Mean \\
\hline $\begin{array}{l}\text { Normalized Variance } \\
\text { (Santos et al., 1997) }\end{array}$ & $\frac{1}{\mathrm{MN}} \sum_{\mathrm{i}=1}^{\mathrm{M}} \sum_{\mathrm{j}=1}^{\mathrm{N}}(\mathrm{LV}(\mathrm{i}, \mathrm{j})-\overline{\mathrm{LV}})^{2}$ \\
\hline $\begin{array}{l}\text { Global Variance based on } \\
\text { Local Variance } \\
\text { (Pech-Pacheco et al., 2000) }\end{array}$ & \multicolumn{1}{c}{$\frac{1}{\mathrm{~B}} \sum_{\mathrm{b}=1}^{\mathrm{B}} 20 \log \frac{\mathrm{G}_{\mathrm{max}, \mathrm{b}}}{\mathrm{G}_{\mathrm{min}, \mathrm{b}}}$} \\
\hline $\begin{array}{l}\text { Measure of Enhancement } \\
\text { (EME) (Agaian et al., 2000) }\end{array}$ & \\
\hline
\end{tabular}




\begin{tabular}{|c|c|}
\hline $\begin{array}{l}\text { Measure of Enhancement by } \\
\text { Entropy (EMEE) } \\
\text { (Agaian et al., 2000) }\end{array}$ & $\frac{1}{B} \sum_{b=1}^{B} \alpha\left(\frac{G_{\max , b}}{G_{\min , b}}\right)^{\alpha} \log \frac{G_{\max , b}}{G_{\min , b}}$ \\
\hline $\begin{array}{l}\text { AME } \\
\text { (Agaian et al., 2007) }\end{array}$ & $\frac{1}{B} \sum_{b=1}^{B} 20 \ln \frac{G_{\max , b}-G_{\min , b}}{G_{\max , b}+G_{\min , b}}$ \\
\hline $\begin{array}{l}\text { AMEE } \\
\text { (Agaian et al., 2007) }\end{array}$ & $\frac{1}{B} \sum_{b=1}^{B} \alpha\left(\frac{G_{\max , b}-G_{\min , b}}{G_{\max , b}+G_{\min , b}}\right)^{\alpha} * \ln \left(\frac{G_{\max , b}-G_{\min , b}}{G_{\max , b}+G_{\min , b}}\right)$ \\
\hline $\begin{array}{l}\text { LogAME } \\
\text { (Wharton et al., 2006) }\end{array}$ & $\frac{1}{\mathrm{~B}} \widetilde{\otimes} \sum_{\mathrm{b}=1}^{\mathrm{B}} 20 \ln \left(\frac{\mathrm{G}_{\max , \mathrm{b}} \widetilde{\Theta} \mathrm{G}_{\min , \mathrm{b}}}{\mathrm{G}_{\max , \mathrm{b}} \widetilde{\oplus} \mathrm{G}_{\min , \mathrm{b}}}\right)$ \\
\hline $\begin{array}{l}\text { LogAMEE } \\
\text { (Wharton et al., 2006) }\end{array}$ & $\frac{1}{\mathrm{~B}} \widetilde{\otimes} \sum_{\mathrm{b}=1}^{\mathrm{B}} \frac{\mathrm{G}_{\mathrm{max}, \mathrm{b}} \widetilde{\Theta} \mathrm{G}_{\min , \mathrm{b}}}{\mathrm{G}_{\mathrm{max}, \mathrm{b}} \widetilde{\oplus} \mathrm{G}_{\min , \mathrm{b}}} * \ln \left(\frac{\mathrm{G}_{\max , \mathrm{b}} \widetilde{\Theta} \mathrm{G}_{\min , \mathrm{b}}}{\mathrm{G}_{\mathrm{max}, \mathrm{b}} \widetilde{\oplus} \mathrm{G}_{\min , \mathrm{b}}}\right)$ \\
\hline $\begin{array}{l}\text { SDME } \\
\text { (Panetta et al., 2011) }\end{array}$ & $-\frac{1}{\mathrm{~B}} \sum_{\mathrm{b}=1}^{\mathrm{B}} 20 \ln \left|\frac{\mathrm{G}_{\max , \mathrm{b}}-2 * \mathrm{G}_{\text {center }, \mathrm{b}}+\mathrm{G}_{\min , \mathrm{b}}}{\mathrm{G}_{\max , \mathrm{b}}+2 * \mathrm{G}_{\text {center }, \mathrm{b}}+\mathrm{G}_{\min , \mathrm{b}}}\right|$ \\
\hline $\begin{array}{l}\text { RME } \\
\text { (Panetta et al., 2013) }\end{array}$ & $\frac{1}{B} \int \sum_{b=1}^{B}\left|\frac{\log \left|G_{\text {center, },}-\overline{G_{b}}\right|}{\log \left|G_{\text {center }, \mathrm{b}}+\overline{\mathrm{G}_{b}}\right|}\right|$ \\
\hline $\begin{array}{l}\text { LocalContrast } \\
\text { (Mittal et al., 2019) }\end{array}$ & $\begin{array}{l}\frac{1}{M N} \sum \sum L C(i, j) \\
\text { Where, } L C(i, j)=\frac{G_{\max , w}-G_{\min , w}}{G_{\max , w}+G_{\min , w}}\end{array}$ \\
\hline FuContrast (Fu \& Shih, 2006) & $\max \left(\frac{\sum_{\mathrm{i}=9}^{15} \text { DCTBand }_{\mathrm{i}}}{\sum_{\mathrm{j}=1}^{8} \text { DCTBand }_{\mathrm{j}}}\right)$ \\
\hline $\begin{array}{l}\text { Average Color Contrast } \\
\text { (ACC) } \\
\text { (Xie \& Wang, 2010) }\end{array}$ & $\begin{array}{c}\frac{1}{(M-1)(N-1)} \sum \sum \mathrm{CC}(\mathrm{i}, \mathrm{j}) \\
\text { Where, } \mathrm{CC}(\mathrm{i}, \mathrm{j})=\frac{1}{\sqrt{3}} \sqrt{\operatorname{GradR}_{\mathrm{i}, \mathrm{j}}^{2}+\mathrm{GradG}_{\mathrm{i}, \mathrm{j}}^{2}+\mathrm{GradB}_{\mathrm{i}, \mathrm{j}}^{2}}\end{array}$ \\
\hline CRME (Panetta et al., 2013) & $\frac{1000}{B} \sqrt{\sum_{b=1}^{B}}\left|\frac{\log \left|G_{\text {center }, b}-\left(\overline{I_{b}}\right)\right|}{\log \left|G_{\text {center }, b}+\left(\overline{I_{b}}\right)\right|}\right|^{\alpha}$ \\
\hline
\end{tabular}

Where, $\mathrm{G}$ is the gray scale image of size $\mathrm{M} \times \mathrm{N} ; \overline{\mathrm{G}}$ is the mean of intensities of the image;

$\overline{\mathrm{LV}}$ is the mean of local variance of all pixels; $G_{\max , b}$ and $G_{\min , b}$ are the highest and lowest value of intensities of $b$ block respectively; $G_{c e n t e r, b}$ represents the pixel value corresponding to the centre pixel; Image is divided into B blocks of size $b_{1} \times b_{2} ; \boldsymbol{\alpha}$ is a constant in the range $[0,1] ; \widetilde{\oplus}, \widetilde{\ominus}, \widetilde{\otimes}$, and $*$ denote the logarithmic image processing (LIP) based operators (Jourlin \& Pinoli, 1988). 


\section{Combined Metrics}

Contrast, colorfulness, brightness, sharpness, etc. are the various factors used for assessing the quality of images. Combined metrics combine two or more individual IQA schemes to determine the overall quality of an image. This subsection details various combined metrics used in the literature.

Fu and Shih (2006) proposed Quality Index (QI) for measuring the quality of color images by incorporating colorfulness, sharpness, and contrast of color images and modelled QI in terms of linear regression. Panetta et al. (2013) explored this technique and presented Color Quality Enhancement (CQE) by using linear relationship among colorfulness, contrast, and sharpness and trained the model using TID2008 database.

Xie and Wang (2010) introduced Color image Assessment Factor (CAF) based on humanspecific image quality parameters by incorporating Average Color Contrast (ACC), Average Information Entropy (AIE), and Normalized Neighborhood Function (NNF) using R, G, and B color channels. As CAF showed more consistency with human visual assessment of images, Sharma and Verma (2017) derived CAF1 by using only ACC and AIE.

Moorthy and Bovik (2010) proposed Support Vector Machine (SVM) based Blind Image Quality Index (BIQI) by incorporating image statistics from distorted images in a two-stage strategy to find the image quality score. The first stage finds the likelihood of the distortion type and the second stage determines the quality score using statistics from distorted images. To find effective features, the given image is applied with wavelet transform on three scales and orientations.

Blind/Referenceless Image Spatial Quality Evaluator (BRISQUE) (Mittal et al., 2011) used SVM based two-stage strategy similar to BIQI to find the quality score, but the algorithm works in the spatial domain and uses a total of 36 features to fit the SVM model.

Natural Image Quality Evaluator (NIQE) (Mittal et al., 2012) is designed in the spatial 
domain using Natural Scene Statistics (NSS). The algorithm uses almost the same NSS features as used by BRISQUE but the NIQE algorithm is trained using the features extracted from natural images of Berkeley image segmentation database by excluding artificially distorted images.

No-Reference Contrast Distorted Image Quality Assessment (NRCDIQA) (Fang et al., 2014) is designed using natural scene statistics and selected first four moment statistics and entropy features of the image to fit its model. The feature vector of NRCDIQA contains the probabilities for a natural image, the first four moment statistics, and entropy.

Table 2. Analysis of combined metrics

\begin{tabular}{|c|c|c|c|c|}
\hline IQA & $\begin{array}{l}\text { Specific } \\
\text { for } \\
\text { Contrast } \\
\text { distortion }\end{array}$ & $\begin{array}{l}\text { Training } \\
\text { Required }\end{array}$ & Training Databases & Testing databases \\
\hline $\mathrm{Fu} \_\mathrm{QI}$ & No & No & - & - \\
\hline CAF & No & No & - & - \\
\hline $\mathrm{CQE}$ & No & Yes & TID2008 & TID2008 \\
\hline CAF1 & No & No & - & - \\
\hline BIQI & No & Yes & LIVE IQA & LIVE IQA \\
\hline BIQME & $\mathrm{No}$ & Yes & Self-generated & 9 databases \\
\hline BRISQUE & No & Yes & LIVE IQA & LIVE IQA \\
\hline CEIQ & Yes & Yes & $\begin{array}{l}\text { CSIQ, } \\
\text { CID2013, CCID2014 }\end{array}$ & $\begin{array}{l}\text { CSIQ, } \\
\text { CID2013, } \\
\text { CCID2014 }\end{array}$ \\
\hline ENIQA & $\mathrm{No}$ & Yes & LIVE IQA & LIVE IQA, TID2013 \\
\hline NIQE & No & Yes & $\begin{array}{l}\text { Copyright-free Flicker } \\
\text { data and Berkeley image } \\
\text { segmentation database }\end{array}$ & LIVE IQA \\
\hline NIQMC & Yes & No & - & $\begin{array}{l}\text { CID2013, } \\
\text { CCID2014, CSIQ, } \\
\text { TID2008, TID2013 }\end{array}$ \\
\hline NRCDIQA & Yes & Yes & SUN2012 & $\begin{array}{l}\text { CID2013, TID2013, } \\
\text { CSIQ }\end{array}$ \\
\hline
\end{tabular}

No-Reference Image Quality Metric for Contrast-distorted (NIQMC) (Gu et al., 2016) utilises local and global details to determine the quality score. The maximum value of entropy of unpredicted regions is used to determine the local details. For the global details, JensenShannon (J-S) divergence was calculated using the Kullback-Leibler divergence by processing image histogram and uniformly distributed histogram with maximum entropy 
value.

Blind Image Quality Measure of Enhanced images (BIQME) (Gu et al., 2017) used 17 features related to contrast, colorfulness, brightness, sharpness, and naturalness of image to predict the quality score. The researchers created more than 100,000 images to train the model and the algorithm is also tested on images from other 9 databases.

Contrast Enhancement based contrast-changed Image Quality (CEIQ) (Yan et al., 2019) method converts the color image into gray scale image and finds its histogram equalized version to determine SSIM, two entropies and two cross-entropy features to model the CEIQ metric.

Entropy-based No-reference Image Quality Assessment (ENIQA) algorithm (Chen et al., 2019) extracts 56 features that span mutual information of RGB channels, mean and skewness of gray scale and sub-band images using joint probability function and two-dimensional entropy by considering the mean value of $8 \times 8$ neighbourhoods in spatial and frequency domain. The details of all approaches can be found in respective papers. Table 2 discusses databases used for training and testing by the combined metrics and whether they are specific to the contrast distortion.

\section{EXPERIMENTAL SETUP}

The empirical simulation analysis of the comparative methods is carried out on MATLAB R2017a software in an Intel i5 laptop with $2.50 \mathrm{GHz}$ speed and 4GB RAM. To analyse the quantitative/visual outcomes of the comparative methods, the simulation analysis is conducted with a large set of synthetic and non-synthetic images with varying textural, brightness, and contrast complexities from different image databases. The performance of various IQA schemes is assessed with benchmarked parameters. This section details the image databases and the assessment criteria used in the comparative study. 


\section{Image Databases}

For comparing the experimental effectiveness of image quality assessment methods in measuring contrast degradations, this study conducted experiments by using contrast-distorted images from CSIQ, TID2013, CCID2014, and PHOS databases. The Computational and Subjective Image Quality (CSIQ) database (Larson \& Chandler, 2010) includes 30 pristine and 116 contrast-distorted images. The image quality ratings are recorded in the form of Difference Mean Opinion Score (DMOS) in the interval [0-1]. The Tempered Image Database 2013 (TID2013) (Ponomarenko et al., 2015) contains 125 images with contrast changes which are selected in this study for evaluating the quality score. The subjective ratings are recorded using the Mean Opinion Score (MOS) in the interval [0-9].

The CCID2014 database (Gu et al., 2015) includes 655 contrast-degraded images from 15 natural scenes. The subjective ratings are recorded in terms of MOS (1-5). The images of CCID2014 are distorted with five types of functions. This study uses images distorted with Gamma transfer, cubic \& logistic, and mean shift functions from CCID2014 database. The PHOS database (Vonikakis et al., 2013) includes 225 color images from 15 scenes of varying illuminations. This database is used in this study to monitor the capability of IQA algorithms to rank the images captured under different illumination conditions.

\section{Evaluation Criteria}

The effectiveness of IQA models is assessed in accordance with their correctness in prediction and prediction monotonicity (Video Quality Experts Group, 2003). As various NR-IQA schemes provide dissimilar quality scores, common benchmarking frameworks are required to assess the effectiveness of various NR-IQA schemes. The previous studies used Pearson Linear Correlation Coefficient (PLCC), Spearman Rank Order Correlation Coefficient (SROCC), Kendall Rank Order Correlation Coefficient (KROCC), Root Mean Squared Error (RMSE), and Average Processing/Computation Time (CT) in seconds as benchmarking 
parameters to assess the effectiveness of various NR-IQA methods.

PLCC and RMSE are utilised for measuring the correctness of the prediction of the IQA model. SROCC and KROCC are used for predicting the monotonicity of the model. KROCC performs better than SROCC in the case where there is no tied value in the data (Puth et al., 2015). In order to compare IQA models, the study uses the Average Spearman's Rank Order Correlation Coefficient (ASROCC) and Average Kendall's Rank Order Correlation Coefficient (AKROCC) to predict the monotonicity of the model. If N, D, and L respectively denote the number of reference images, degradation types, and distortion levels, the ASROCC and AKROCC are respectively defined as

$$
\begin{aligned}
& \operatorname{ASROCC}=\frac{1}{\mathrm{D}} \sum_{\mathrm{i}=1}^{\mathrm{D}} \mathrm{abs}\left(\frac{1}{\mathrm{~N}} \sum_{\mathrm{j}=1}^{\mathrm{N}} \operatorname{SROCC}\left(\mathrm{Q}_{\mathrm{ij}}, \mathrm{S}_{\mathrm{ij}}\right)\right) \\
& \mathrm{AKROCC}=\frac{1}{\mathrm{D}} \sum_{\mathrm{i}=1}^{\mathrm{D}} \mathrm{abs}\left(\frac{1}{\mathrm{~N}} \sum_{\mathrm{j}=1}^{\mathrm{N}} \operatorname{KROCC}\left(\mathrm{Q}_{\mathrm{ij}}, \mathrm{S}_{\mathrm{ij}}\right)\right)
\end{aligned}
$$

Where, $S_{i j}$ and $Q_{i j}$ are vectors of length $L$ respectively showing the quality scores provided by the IQA scheme and the distortion levels corresponding to the given set of distorted images of $\mathrm{j}$-th source image with $\mathrm{i}$-th functions used to generate the distorted images. Though AKROCC values are also analysed, these values are not presented in the paper due to the want of space.

\section{EXPERIMENTAL RESULTS AND ANALYSIS}

To analyse IQA metrics, the fidelity of the image is considered so that future applications can choose the correct metric while using these metrics to check the performance of the contrast enhancement schemes. This section discusses the empirical results of colorfulness, contrast, and combined metrics corresponding to various distorted images and makes the comparative analysis of these methods in terms of their performance with actual results. 


\section{Analysis for colorfulness Metrics}

To measure the colorfulness of color images, the study compares five metrics. The average processing time (APT) of all five colorfulness-based algorithms are measured from 116 contrast-distorted images with size $512 \times 512 \times 3$ of CSIQ database and tabulated in Table 3 . Mean_Saturation and CQE_Colorfulness ${ }_{1}$ are the best two algorithms in terms of average runtime. From Fig. 1(a), it is visible that the average processing time of CQE_Colorfulness 1 and QI_Colorfulness are almost the same. It is noted that the Colorfulness Index requires more time for converting RGB images to CIELUV color space.

Table 3. APT of colorfulness metrics on 116 images of CSIQ database

\begin{tabular}{|l|l|l|l|}
\hline Metric Code & Colorfulness Metric & APT (in Sec.) & Rank \\
\hline C1 & Colorfulness Index & 0.0630 & 5 \\
\hline C2 & QI_Colorfulness & 0.0123 & 3 \\
\hline C3 & CQE_Colorfulness ${ }_{1}$ & 0.0122 & 2 \\
\hline C4 & CQE_Colorfulness 2 & 0.0145 & 4 \\
\hline C5 & Mean_Saturation & 0.0046 & 1 \\
\hline
\end{tabular}

Table 4 shows the ASROCC values of different colorfulness metrics corresponding to distorted images from different databases. From Table 4, Fig. 1(b), and Fig. 1(c), it can be easily assessed that the CQE_Colorfulness 2 shows the least performance as its ASROCC is the lowest when compared to other metrics.

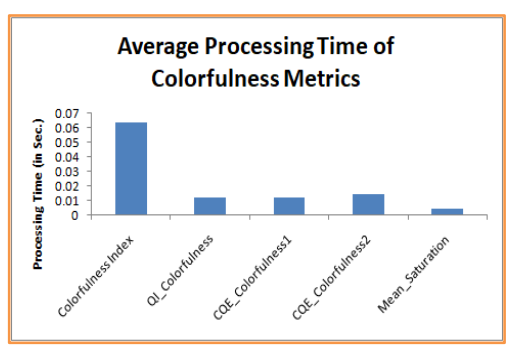

(a)

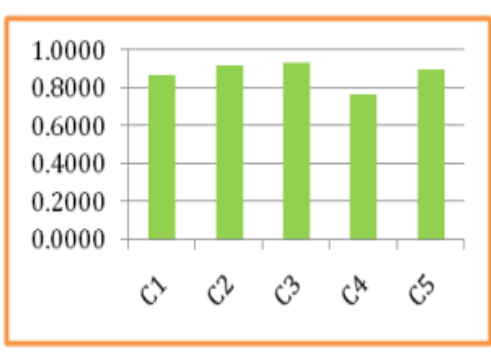

(b)

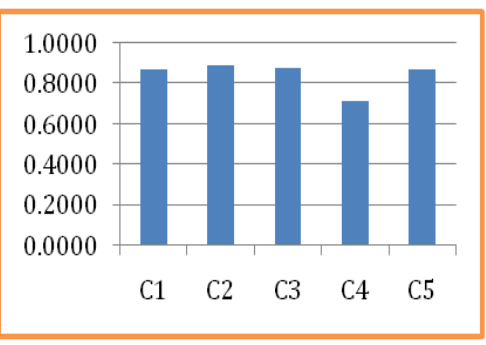

(c)

Figure 1. Results of colorfulness metrics (a) Average processing time of colorfulness metrics (b) ASROCC direct average values of colorfulness metrics (c) Database size-weighted average values of ASROCC of colorfulness metric

The databases used in this comparative study provide contrast distorted images generated using different functions and hence the effects of all metrics on these different functions are measured separately. CSIQ and TID2013 databases have used only one function to generate 
the distorted images with contrast change. In both cases, all metrics except

CQE_Colorfulness ${ }_{2}$ follow the distortion level and visual ranking as shown in Table 4.

Table 4. ASROCC values of colorfulness metrics

\begin{tabular}{|l|l|c|c|c|c|c|}
\hline \multirow{2}{*}{$\begin{array}{l}\text { Data } \\
\text { base }\end{array}$} & Type of Distortion & \multicolumn{6}{|c|}{ Colorfulness Metrics } \\
\cline { 3 - 7 } & & $\mathbf{C 1}$ & $\mathbf{C 2}$ & $\mathbf{C 3}$ & $\mathbf{C 4}$ & $\mathbf{C 5}$ \\
\hline CSIQ & Contrast Change-ASROCC & $\mathbf{1 . 0 0}$ & $\mathbf{1 . 0 0}$ & $\mathbf{1 . 0 0}$ & $\mathbf{0 . 8 9}$ & $\mathbf{1 . 0 0}$ \\
\hline \multirow{3}{*}{ PHOS } & Non Uniform & 0.16 & 0.56 & 0.89 & 0.29 & 0.92 \\
\cline { 2 - 7 } & Uniform-Dark & 0.53 & 1.00 & 1.00 & 0.73 & 0.31 \\
\cline { 2 - 7 } & Uniform-Bright & 1.00 & 0.88 & 0.92 & 0.72 & 1.00 \\
\cline { 2 - 7 } & ASROCC & $\mathbf{0 . 5 7}$ & $\mathbf{0 . 8 1}$ & $\mathbf{0 . 9 4}$ & $\mathbf{0 . 5 8}$ & $\mathbf{0 . 7 4}$ \\
\hline \multirow{2}{*}{ TID2013 } & Contrast Change-ASROCC & $\mathbf{1 . 0 0}$ & $\mathbf{1 . 0 0}$ & $\mathbf{1 . 0 0}$ & $\mathbf{0 . 9 2}$ & $\mathbf{0 . 9 9}$ \\
\hline \multirow{3}{*}{2014} & Gamma Transfer & 1.00 & 0.83 & 0.84 & 0.68 & 1.00 \\
\cline { 2 - 7 } & Cubic and Logistic & & & & & \\
& Function & 1.00 & 0.79 & 0.73 & 0.84 & 1.00 \\
\cline { 2 - 7 } & Mean Shift & 0.78 & 0.99 & 0.88 & 0.55 & 0.61 \\
\cline { 2 - 7 } & ASROCC & $\mathbf{0 . 9 3}$ & $\mathbf{0 . 8 7}$ & $\mathbf{0 . 8 2}$ & $\mathbf{0 . 6 9}$ & $\mathbf{0 . 8 7}$ \\
\hline ASROCC Direct Average & 0.87 & 0.92 & 0.94 & 0.77 & 0.90 \\
\hline \multicolumn{2}{|l|}{ Database } & size-weighted average & 0.89 & 0.88 & 0.72 & 0.87 \\
\hline
\end{tabular}

Natural images are usually prone to uneven illumination and hence it is advisable to use Mean_Saturation and CQE_Colorfulness 1 as their ASROCC values in non-uniformly illuminated images from the PHOS database are higher than other algorithms. The ASROCC of Mean_Saturation and Colorfulness Index is 1 in uniformly bright images for the PHOS database so it is advisable to use Mean_Saturation while working with bright images.

In the case of dark images, CQE_Colorfulness1 and QI_Colorfulness are showing better results than other schemes. By analysing various IQA metrics under different image conditions through Table 3-4 and Fig. 1(a, b, c), it is observed that CQE_Colorfulness 1, QI_Colorfulness, and Mean_Saturation are the best methods for measuring the colorfulness of the given image under different situations.

\section{Analysis for Contrast Metrics}

The study performs the experimental comparison of 17 algorithms to demarcate their performance in measuring the contrast of images in different situations. The gray scale metrics Range, variance, RMSC, and color image metrics- ACC recorded the lowest average 
processing time as shown in Fig. 2(a) and Table 5.

Table 5. Average processing time (APT) of contrast metrics on 116 images of CSIQ database

\begin{tabular}{|l|l|l|l|l|l|l|}
\hline Metric Code & CT1 & CT2 & CT3 & CT4 & CT5 & CT6 \\
\hline Contrast Metric & AME & AMEE & EME & EMEE & LogAME & LogAMEE \\
\hline APT (in sec.) & 0.0362 & 0.0369 & 0.0334 & 0.0341 & 0.0393 & 0.0395 \\
\hline Metric Code & CT7 & CT8 & CT9 & CT10 & CT11 & CT12 \\
\hline Contrast Metric & SDME & RME & Variance & Range & NormVar & RMSC \\
\hline APT (in sec.) & 0.1158 & 0.0309 & 0.0024 & 0.0003 & 0.0026 & 0.0014 \\
\hline Metric Code & CT13 & CT14 & CT15 & CT16 & CT17 & \\
\hline Contrast Metric & ACC & LocalContrast & GlobalVar & CRME & FuContrast & \\
\hline APT (in sec.) & 0.0669 & 0.1324 & 0.0142 & 0.3444 & 0.1508 & \\
\hline
\end{tabular}

From Fig. 2 and Table 6, it is identified that though most of the metrics show good performance in specific situations, very few algorithms are stable in their performance in all situations. As it is seen from Table 6, AME and AMEE are good in all situations except for images degraded with uniform increased brightness. LogAME and LogAMEE are good only for contrast changes, gamma transfer, and non-uniform images. SDME is good only for images degraded with contrast change and gamma transfer functions. RME is not suitable for gamma transfer and uniform-dark images while LocalContrast is not good for uniform-bright images. Range is highly influenced by the outliers and hence it can't be a good measure.

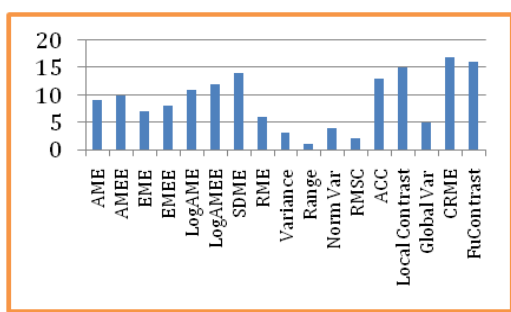

(a)

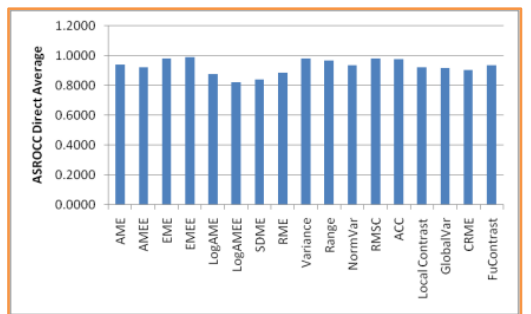

(b)

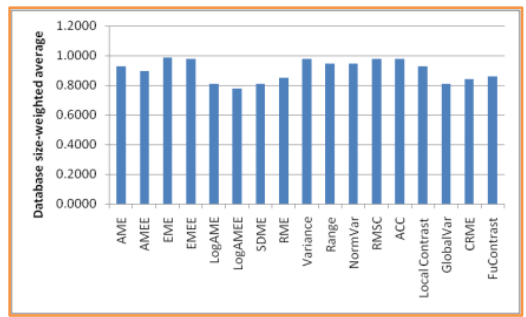

(c)

Figure 2. Results of contrast metrics (a) Rank of average processing time of contrast metrics on 116 images of CSIQ database (b) ASROCC direct average values of contrast metrics (c) Database size-weighted average values of ASROCC of contrast metrics

In Fig. 3, there are six images of uneven illumination from the PHOS database. It is seen that the brightness of images is monotonically decreasing with the last image as the darkest. Though it is expected from the metrics that these algorithms should follow the same order either in positive (ascending order) or in negative (descending order) direction, LogAMEE, 
SDME, RME, RMSC, GlobalVar, and CRME are not following the same order as shown in

Table 7.

Table 6. ASROCC values of contrast metrics

\begin{tabular}{|c|c|c|c|c|c|c|c|c|c|c|c|c|}
\hline \multirow{2}{*}{$\begin{array}{l}\text { Data } \\
\text { base }\end{array}$} & \multirow{2}{*}{\begin{tabular}{ll|} 
Type of \\
Distortion
\end{tabular}} & \multicolumn{11}{|c|}{ Contrast Metrics } \\
\hline & & CT1 & CT2 & CT & & $\mathrm{CT}$ & & T5 & $\mathrm{CT}$ & CT7 & CT8 & CT9 \\
\hline CSIQ & $\begin{array}{l}\text { Contrast Change- } \\
\text { ASROCC }\end{array}$ & 0.99 & 0.99 & 1.0 & & 1.0 & & 98 & 0.9 & 0.97 & 1.00 & 1.00 \\
\hline \multirow[t]{4}{*}{ PHOS } & Non Uniform & 1.00 & 1.00 & 1.0 & & 1.0 & & 00 & 0.9 & 0.31 & 0.97 & 0.86 \\
\hline & Uniform-Dark & 1.00 & 1.00 & 1.0 & & 0.99 & & 79 & 0.0 & 0.62 & 0.17 & 0.99 \\
\hline & Uniform-Bright & 0.49 & 0.47 & 1.0 & & 1.0 & & 49 & 0.5 & 0.81 & 1.00 & 1.00 \\
\hline & Average SROCC & $\mathbf{0 . 8 3}$ & 0.82 & 1.0 & & 1.0 & & 76 & 0.5 & 0.58 & 0.71 & 0.95 \\
\hline $\begin{array}{l}\text { TID } \\
2013\end{array}$ & $\begin{array}{l}\text { Contrast Change - } \\
\text { ASROCC }\end{array}$ & 1.00 & 1.00 & 0.9 & & 1.0 & & 00 & 1.0 & 0.99 & 1.00 & 1.00 \\
\hline \multirow{4}{*}{$\begin{array}{l}\text { CCID } \\
2014\end{array}$} & Gamma Transfer & 0.93 & .97 & 0 & & .93 & & 3 & 1.0 & 0.97 & 0.52 & 0.93 \\
\hline & $\begin{array}{l}\text { Cubic and } \\
\text { Logistic Function }\end{array}$ & 0.91 & 0.86 & 1.0 & & 1.0 & & 8 & 0.8 & 0.67 & 0.99 & 1.00 \\
\hline & Mean Shift & 0.95 & 0.81 & 0.9 & & 0.9 & & 57 & 0.5 & 0.84 & 1.00 & 1.00 \\
\hline & Average SROCC & 0.93 & 0.88 & 0.9 & & 0.9 & & 76 & 0.7 & 0.83 & 0.84 & 0.98 \\
\hline \multicolumn{2}{|c|}{ ASROCC Direct Average } & 0.94 & 0.92 & 0.9 & & 0.9 & & 88 & 0.8 & 0.84 & 0.89 & 0.98 \\
\hline \multicolumn{2}{|c|}{$\begin{array}{l}\text { Database size-weighted } \\
\text { average }\end{array}$} & 0.93 & 0.89 & 0.9 & & 0.98 & & 81 & 0.7 & 0.81 & 0.85 & 0.98 \\
\hline $\begin{array}{l}\text { Data } \\
\text { base }\end{array}$ & Type of Distortion & CT10 & CT11 & & CT1 & & 13 & & & T15 & $\mathrm{T} 16$ & CT17 \\
\hline CSIQ & $\begin{array}{l}\text { Contrast Change- } \\
\text { ASROCC }\end{array}$ & 10 & 100 & & 1.00 & & 1.00 & & 0 & 1.00 & 1.00 & 1.00 \\
\hline \multirow{4}{*}{ PHOS } & form & o & 0 & & 0 & & & & & 0.90 & 0.69 & 0.98 \\
\hline & Uniform-Dark & 0.98 & 0.24 & & 0.99 & & 1.00 & & .00 & 1.00 & 0.91 & 0.99 \\
\hline & Uniform-Bright & 1.00 & 1.00 & & 1.00 & & 1.00 & & .12 & 0.99 & 1.00 & 0.99 \\
\hline & SROCC & 0.99 & $\mathbf{0 . 7 5}$ & & 0.95 & & 2 & & 71 & 0.99 & 0.86 & 0.99 \\
\hline $\begin{array}{l}\text { TID } \\
2013\end{array}$ & $\begin{array}{l}\text { Contrast Change - } \\
\text { ASROCC }\end{array}$ & 0.98 & 1.00 & & 1.00 & & 1.00 & & .00 & 1.00 & 0.98 & 1.00 \\
\hline \multirow{4}{*}{$\begin{array}{l}\text { CCID } \\
2014\end{array}$} & Gamma Transfer & 0.81 & 0.98 & & 0.93 & & 0.97 & & .00 & 0.35 & 0.82 & 1.00 \\
\hline & $\begin{array}{l}\text { Cubic and Logistic } \\
\text { Function }\end{array}$ & 0.93 & 1.00 & & 1.00 & & 1.00 & & .96 & 0.69 & 0.67 & 0.74 \\
\hline & Mean Shift & 1.00 & 0.99 & & 1.00 & & 1.00 & & .97 & 1.00 & 0.85 & 0.93 \\
\hline & Average SROCC & 0.91 & 0.99 & & 0.98 & & 0.99 & & .98 & 0.68 & 0.78 & 0.89 \\
\hline \multicolumn{2}{|c|}{ ASROCC Direct Average } & 0.97 & 0.93 & & 0.98 & & 0.98 & & .92 & 0.92 & 0.91 & 0.97 \\
\hline \multicolumn{2}{|c|}{$\begin{array}{c}\text { Database size-weighted } \\
\text { average }\end{array}$} & 0.94 & 0.9 & & 0.98 & & 0.98 & & .93 & 0.81 & 0.84 & 0.93 \\
\hline
\end{tabular}

Overall EME, EMEE, Variance, RMSC, and ACC are good measures of contrast variations as their ASROCC direct average value is better than other measures as can be visualised from Fig. 2(b). The weighted average ASROCC values of EME among gray scale metrics and of ACC from color image metrics are the best ones as shown in Fig. 2(c). 


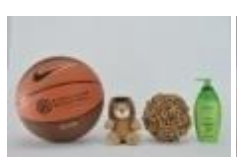

(a)

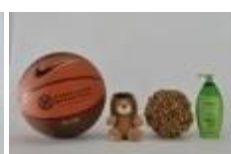

(b)

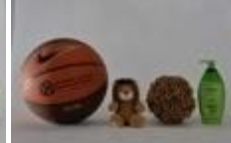

(c)

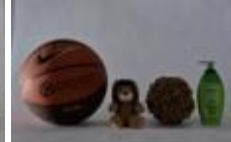

(d)

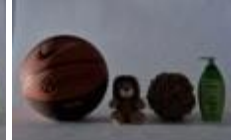

(e)

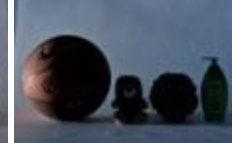

(f)

Figure 3. Non-uniform illuminated images from scene 1 of the PHOS database.

Table 7. Contrast metrics' values for the images shown in Fig. 3.

\begin{tabular}{|l|c|c|c|c|c|c|}
\hline Contrast Metric & Fig. 3(a) & Fig. 3(b) & Fig. 3(c) & Fig. 3(d) & Fig. 3(e) & Fig. 3(f) \\
\hline AME & 35.7585 & 34.5158 & 30.5003 & 27.6019 & 26.2412 & 24.0672 \\
AMEE & 0.0804 & 0.0834 & 0.0927 & 0.1000 & 0.1035 & 0.1088 \\
EME & 1.3176 & 1.6171 & 2.0919 & 2.5337 & 2.9562 & 3.7772 \\
EMEE & 0.1508 & 0.2244 & 0.3702 & 0.6169 & 1.0167 & 1.6446 \\
LogAME & 0.0942 & 0.0934 & 0.0865 & 0.0822 & 0.0813 & 0.0811 \\
LogAMEE & 0.0419 & 0.0433 & 0.0471 & 0.0486 & 0.0482 & 0.0462 \\
SDME & 59.4715 & 61.3743 & 60.8814 & 57.5974 & 56.5093 & 52.6209 \\
RME & 1.9448 & 1.9958 & 2.0825 & 2.1337 & 2.1430 & 2.1292 \\
Variance & 37.8357 & 37.3179 & 39.8988 & 44.6497 & 48.2404 & 55.2027 \\
Range & 233.0000 & 242.0000 & 250.0000 & 253.0000 & 254.0000 & 255.0000 \\
NormVar & 7.8829 & 8.9940 & 12.2405 & 17.4922 & 22.2120 & 31.5138 \\
RMSC & 0.1484 & 0.1463 & 0.1565 & 0.1751 & 0.1892 & 0.2165 \\
ACC & 3.0817 & 2.9329 & 2.8302 & 2.6723 & 2.5767 & 2.4607 \\
LocalContrast & 0.0492 & 0.0594 & 0.0738 & 0.0873 & 0.0992 & 0.1279 \\
GlobalVar & 256.8739 & 235.0068 & 194.3621 & 175.0702 & 176.7542 & 202.8071 \\
CRME & 1.4253 & 1.4507 & 1.4499 & 1.3411 & 1.3616 & 1.4444 \\
FuContrast & 0.1842 & 0.2312 & 0.2512 & 0.2903 & 0.3003 & 0.4660 \\
\hline
\end{tabular}

\section{Analysis for Combined Metrics}

This section analyses the performance of 12 combined metrics including 9 general-purpose and 3 contrast distortion specific IQA schemes. From Table 8 and Fig. 4(a), it is observed that the average processing time of CEIQ, CAF1, CAF, and NRCDIQA are the lowest when compared to other combined measures used in the comparative study.

Table 8. Average processing time (APT) of combined metrics on 116 images of CSIQ database

\begin{tabular}{|l|c|c|c|c|c|c|}
\hline Combined Metrics & $\mathrm{Fu} \_\mathrm{QI}$ & $\mathrm{CAF}$ & $\mathrm{CQE}$ & $\mathrm{CAF} 1$ & CEIQ & NRCDIQA \\
\hline APT (in Sec.) & 0.1851 & 0.0725 & 0.3265 & 0.0663 & 0.0451 & 0.1007 \\
\hline Combined Metrics & $\mathrm{BRISQUE}$ & $\mathrm{BIQI}$ & NIQMC & NIQE & ENIQA & BIQME \\
\hline APT (in Sec.) & 0.2470 & 0.7509 & 1.7079 & 0.1915 & 6.6777 & 0.7128 \\
\hline
\end{tabular}

From Table 9, it is clear that most of the general-purpose algorithms including CAF, BRISQUE, BIQI, NIQE, and ENIQA are not working effectively in the case of contrastdistorted images as their ASROCC direct average values are less than 0.8. Only Fu_QI and 
BIQME produce ASROCC direct average values in the range of 0.8 to 0.9 . Further, it is clear from the experimental analysis that CAF1 and CQE among general-purpose algorithms produce ASROCC direct average value greater than 0.9. CAF1 is the best since CQE requires more processing time than CAF1.

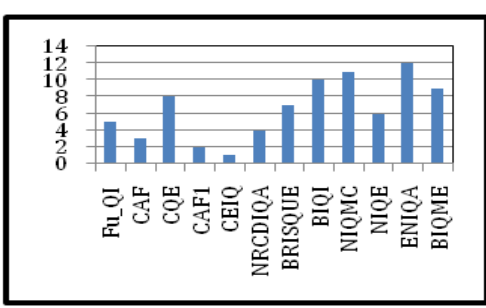

(a)

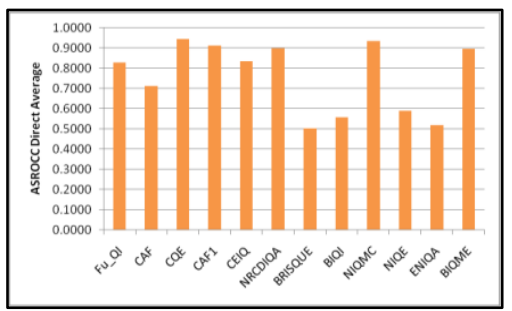

(b)

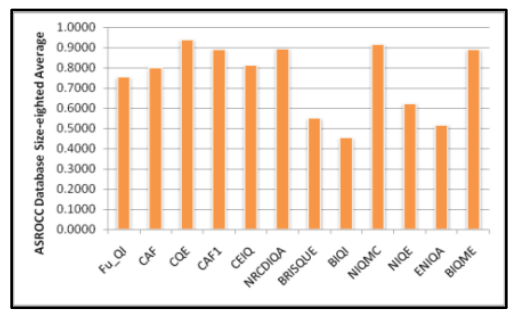

(c)

Figure 4. Results of combined metrics (a) Rank in ascending order of processing time of combined metrics (b) Direct average of ASROCC values of different combined metrics (c) Database size-weighted average of ASROCC values of different combined metrics

From Fig. 4(b), it is further seen that NIQMC and NRCDIQA among contrast-distorted metrics produce better results as their respective ASROCC direct average values are above 0.9. NIQMC requires almost 16 times the processing time of NRCDIQA to process an image.

From Fig. 4(c), it is assessed that CQE outperforms all other combined metrics as its database size-weighted average ASROCC values are higher.

Table 9. ASROCC Values of Combined Metrics

\begin{tabular}{|c|c|c|c|c|c|c|c|}
\hline \multirow[t]{2}{*}{ Database } & \multirow[t]{2}{*}{ Type of Distortion } & \multicolumn{6}{|c|}{ Combined Metrics } \\
\hline & & $\mathrm{Fu} \_\mathrm{QI}$ & CAF & CQE & CAF1 & CEIQ & $\begin{array}{l}\text { NRCD } \\
\text { IQA }\end{array}$ \\
\hline CSIQ & $\begin{array}{l}\text { Contrast Change- } \\
\text { ASROCC }\end{array}$ & 1.00 & 0.76 & 1.00 & 1.00 & 0.99 & 0.95 \\
\hline \multirow[t]{4}{*}{ PHOS } & Non Uniform & 0.35 & 0.52 & 0.83 & 0.90 & 0.94 & 1.00 \\
\hline & Uniform-Dark & 0.51 & 0.38 & 0.89 & 0.99 & 0.27 & 0.99 \\
\hline & Uniform-Bright & 0.92 & 1.00 & 1.00 & 1.00 & 0.61 & 1.00 \\
\hline & ASROCC & 0.59 & 0.63 & 0.91 & 0.97 & 0.61 & 1.00 \\
\hline TID2013 & $\begin{array}{l}\text { Contrast Change- } \\
\text { ASROCC }\end{array}$ & 1.00 & 0.55 & 0.94 & 0.84 & 0.91 & 0.79 \\
\hline \multirow[t]{4}{*}{ CCID2014 } & Gamma Transfer & 0.80 & 1.00 & 0.90 & 1.00 & 1.00 & 0.96 \\
\hline & $\begin{array}{l}\text { Cubic and Logistic } \\
\text { Function }\end{array}$ & 0.65 & 0.74 & 0.95 & 0.57 & 0.50 & 0.65 \\
\hline & Mean Shift & 0.70 & 1.00 & 0.97 & 1.00 & 1.00 & 0.99 \\
\hline & ASROCC & 0.72 & 0.91 & 0.94 & 0.86 & $\mathbf{0 . 8 3}$ & 0.87 \\
\hline \multicolumn{2}{|c|}{ ASROCC Direct Average } & 0.83 & 0.71 & 0.95 & 0.91 & 0.84 & 0.90 \\
\hline \multicolumn{2}{|c|}{ Database size-weighted average } & 0.75 & 0.80 & 0.94 & 0.89 & 0.81 & 0.89 \\
\hline
\end{tabular}




\begin{tabular}{|c|c|c|c|c|c|c|c|}
\hline Database & Type of Distortion & $\begin{array}{l}\text { BRIS } \\
\text { QUE }\end{array}$ & BIQI & NIQMC & NIQE & $\begin{array}{l}\text { ENI } \\
\text { QA }\end{array}$ & BIQME \\
\hline CSIQ & $\begin{array}{l}\text { Contrast Change- } \\
\text { ASROCC }\end{array}$ & 0.17 & $\mathbf{0 . 8 3}$ & 1.00 & 0.69 & 0.67 & 0.94 \\
\hline \multirow[t]{4}{*}{ PHOS } & Non Uniform & 0.96 & 0.25 & 0.62 & 0.10 & 0.43 & 0.77 \\
\hline & Uniform-Dark & 0.61 & 0.79 & 1.00 & 0.77 & 0.96 & 0.71 \\
\hline & Uniform-Bright & 0.99 & 0.55 & 1.00 & 0.93 & 0.28 & 0.83 \\
\hline & ASROCC & 0.85 & 0.53 & 0.87 & 0.60 & 0.56 & 0.77 \\
\hline TID2013 & $\begin{array}{l}\text { Contrast Change- } \\
\text { ASROCC }\end{array}$ & 0.46 & 0.53 & 0.97 & 0.40 & $\mathbf{0 . 3 3}$ & 0.97 \\
\hline \multirow[t]{4}{*}{ CCID2014 } & Gamma Transfer & 0.18 & 0.38 & 0.99 & 0.69 & 0.71 & 1.00 \\
\hline & $\begin{array}{l}\text { Cubic and Logistic } \\
\text { Function }\end{array}$ & 0.65 & 0.28 & 0.72 & 0.63 & 0.54 & 0.74 \\
\hline & Mean Shift & 0.76 & 0.38 & 1.00 & 0.67 & 0.29 & 0.99 \\
\hline & ASROCC & 0.53 & 0.35 & 0.90 & 0.66 & 0.51 & 0.91 \\
\hline \multicolumn{2}{|c|}{ ASROCC Direct Average } & 0.50 & 0.56 & 0.94 & 0.59 & 0.52 & 0.90 \\
\hline \multicolumn{2}{|c|}{ Database size-weighted average } & 0.55 & 0.45 & 0.92 & 0.62 & 0.52 & 0.89 \\
\hline
\end{tabular}

\section{CONCLUSION}

This study provided an insight into major general-purpose NR-IQA methods and their effectiveness in assessing contrast, colorfulness, and overall quality of contrast-degraded images with technical analysis. The paper analysed the effectiveness of top-ranking NR-IQA methods with benchmark assessment methods on images from standard image databases. The study finds that under different situations, different IQA schemes are good to assess image quality. From the experimental analysis conducted with numerous contrast distorted images from various databases, the study identifies that QI_Colorfulness and CQE_colorfulness ${ }_{1}$ are better to measure colorfulness while ACC and EME are good for measuring contrast. Further, the study finds that the CQE and CAF1 among general-purpose metrics and NIQMC and NRCDIQA among contrast distorted metrics are good to assess the overall image quality.

\section{FUTURE DiRECTIONS}

NR-IQA faces huge challenges because of the technical problems associated with qualityaware feature extraction and thereby effectively designing models that produce quality scores in accordance with the human subjective analysis. Although there is significant progress in 
the field of NR-IQA, there is scope for improvements.

\section{Computational inefficiency and erroneous features}

NR-IQA usually works in feature extraction and quality score generation phases. Most of the NR-IQAs such as BRISQUE, BIQI, NIQE, and ENIQA take substantial computation time in extracting features but didn't work well in case of contrast distorted images. NIQMC uses a linear combination of features with a constant weight. The ASROCC of NIQMC is good in terms of its results but its performance can be improved in the future if the weights are made adaptive. CAF1 is designed based on contrast and entropy features but the colorfulness feature is excluded. NRCDIQA used NSS with respect to the moment and entropy characteristics of the input image.

The metrics work either directly on R, G, B channels or on other color channels such as the $\mathrm{Y}$ channel in $\mathrm{YCbCr}$, and the $\mathrm{V}$ channel in HSV color space. These channels also influence the performance of algorithms (Bezryadin et al., 2007). So in the future, the algorithms can try selecting effective features of contrast distorted images by performing dimensionality reduction to reduce computational cost.

\section{Reliance on Subjective Score}

Most of the NR-IQAs are opinion aware metrics that are trained and tested using the subjective score of images. The subjective score reflects the perceived quality of the image and it depends on the stimuli used while recording the score by the individual subject. It is harder for individuals to provide accurate scores that suit the visual quality of images under consideration. Further, the subjective scores do not be exact with respect to the visual quality of images. It can be seen from Fig. 5 that the MOS outputs of images are not correlating with the distortion levels and it leads to non-reliability of estimation models. The human judgements are ambiguous in nature and hence the subjective score in the future can look to record subjective scores with fuzzy scale values rather than with crisp values. 


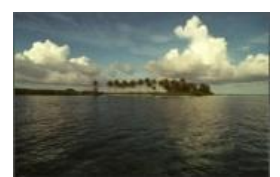

(a) $\mathrm{MOS}=3.2324$, Rank $=1$

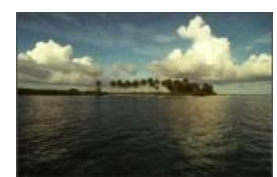

(b) $\mathrm{MOS}=2.8898$, Rank=2

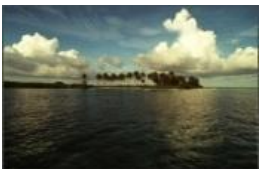

(c) MOS=2.9140, Rank=3

Figure 5. Three images distorted using the logistic function from CCID2014 database with ranks and MOS Values

\section{Reliance on Database}

It is seen from the tabulated values of Table 2 and Table 9 that the competing schemes provide effective results if the training and testing images are performed on similar distortions such as in the case of NRCDIQA. From general-purpose algorithms, CQE provides better performance since it is trained and tested with contrast change images. But it is observed that BRISQUE, BIQI, ENIQA, NIQE, and ENIQA fail in providing correct assessment due to the absence of training with contrast distorted images. This indicates the need for competing NRIQA algorithms to be altered and trained for adapting their performance to new distortions.

\section{REFERENCES}

Agaian, S.S., Lentz, K.P. \& Grigoryan, A.M., 2000. A new measure of image enhancement. Proceedings of IASTED International Conference on Signal Processing \& Communication, Malaga.

Agaian, S.S., Silver, B. \& Panetta, K.A., 2007. Transform coefficient histogram-based image enhancement algorithms using contrast entropy. IEEE transactions on image processing, 16(3): 741-758.

Ahmed, I.T., Der, C.S. \& Hammad, B.T., 2017. Recent approaches on no-reference image quality assessment for contrast distortion images with multiscale geometric analysis transforms: A survey. Journal of Theoretical \& Applied Information Technology, 95(3): $561-569$.

Bezryadin, S., Bourov, P. \& Ilinih, D., 2007. Brightness calculation in digital image processing. Proceedings of $\mathrm{I}^{\text {st }}$ International symposium on technologies for digital photo fulfillment, Las Vegas. 
Bosse, S., Maniry, D., Wiegand, T. \& Samek, W., 2016. A deep neural network for image quality assessment. Proceedings of 2016 IEEE International Conference on Image Processing (ICIP), Phoenix, USA.

Bouzerdoum, A., Havstad, A. \& Beghdadi, A., 2004. Image quality assessment using a neural network approach. Proceedings of the Fourth IEEE International Symposium on Signal Processing and Information Technology, Rome, Italy.

Chen, X., Zhang, Q., Lin, M., Yang, G. \& He, C. 2019. No-reference color image quality assessment: from entropy to perceptual quality. EURASIP Journal on Image and Video Processing, 2019: 77.

Ding, K., Ma, K., Wang, S. \& Simoncelli, E.P., 2021. Comparison of Full-Reference image quality models for optimization of image processing systems. International Journal of computer vision, 129: 1258-1281.

Eskicioglu, A.M. \& Fisher, P.S., 1995. Image quality measures and their performance. IEEE Transactions on Communications, 43(12): 2959-2965.

Fang, Y., Ma, K., Wang, Z., Lin, W., Fang, Z. \& Zhai, G., 2014. No-reference quality assessment of contrast-distorted images based on natural scene statistics. IEEE Signal Processing Letters, 22(7): 838-842.

Firestone, L., Cook, K., Culp, K., Talsania, N. \& Preston K. Jr, 1991. Comparison of autofocus methods for automated microscopy. Cytometry, 12(3): 195-206.

Fu, Y.-Y. \& Shih, F.Y., 2006. Color image quality measures and retrieval. Ph.D. dissertation, New Jersey Institute of Technology, New Jersey, United States.

Gao, C., Panetta, K. \& Agaian, S., 2014. Color image attribute and quality measurements. Proceedings SPIC 9120, Mobile Multimedia/Image Processing, Security, and Applications, Baltimore, Maryland, USA. 
George, A.G. \& Prabavathy, K., 2014. A survey on different approaches used in image quality assessment. International Journal of Computer Science and Network Security, 14(2): 78-84.

Gonzalez, R.C. \& Woods, R.E., 2006. Digital Image Processing, Pearson Education, London.

Gu, K., Lin, W., Zhai, G., Yang, X., Zhang, W. \& Chen, C.W., 2016. No-reference quality metric of contrast-distorted images based on information maximization. IEEE Transactions on Cybernetics, 47(12): 4559-4565.

Gu, K., Tao, D., Qiao, J.-F. \& Lin, W., 2017. Learning a no-reference quality assessment model of enhanced images with big data. IEEE Transactions on neural networks and learning systems, 29(4): 1301-1313.

Gu, K., Zhai, G., Lin, W. \& Liu, M., 2015. The analysis of image contrast: From quality assessment to automatic enhancement. IEEE Transactions on Cybernetics, 46(1): 284-297.

Hasler, D. \& Suesstrunk, S.E., 2003. Measuring colorfulness in natural images. Proceedings SPIC 5007, Human vision and electronic imaging VIII, Santa Clara, California.

Jourlin, M. \& Pinoli, J.-C., 1988. A model for logarithmic image processing. Journal of microscopy, 149(1): 21-35.

Kamble, V. \& Bhurchandi, K.M., 2015. No-reference image quality assessment algorithms: A survey. Optik, 126(11-12): 1090-1097.

Krishnendu, M., Priya, M. \&Joby, J., 2021. No-Reference Image Quality Analysis-An Overview. International Journal of Engineering Research \& Technology, 9(13): 56-59.

Krotkov, E. \& Martin, J.-P., 1986. Range from focus. Proceedings of 1986 IEEE International Conference on Robotics and Automation, San Francisco, California.

Larson, E.C. \& Chandler, D.M., 2010. Most apparent distortion: full-reference image quality assessment and the role of strategy. Journal of electronic imaging, 19(1): 011006. 
Li, C., Bovik, A.C. \& Wu, X., 2011. Blind Image Quality Assessment Using a General Regression Neural Network. IEEE Transactions on Neural Networks, 22(5): 793-799.

Liu, X., Weijer, J.V.D. \& Bagdanov, A.D., 2017. RankIQA: Learning from Rankings for No-Reference Image Quality Assessment. Proceedings of 2017 IEEE International Conference on Computer Vision (ICCV), Venice, Italy.

Ma, K. et al., 2017. dipIQ: Blind image quality assessment by learning-to-rank discriminable image pairs. IEEE Transactions on Image Processing, 26(8): 3951-3964.

Manap, R.A. \& Shao, L., 2015. Non-distortion-specific no-reference image quality assessment: A survey. Information Sciences, 301: 141-160.

Mittal, A., Moorthy, A.K. \& Bovik, A.C., 2011. Blind/referenceless image spatial quality evaluator. Conference record of the forty fifth Asilomar conference on signals, systems and computers, Pacific Grove, California.

Mittal, P., Saini, R.K. \& Jain, N.K., 2019. Image enhancement using fuzzy logic techniques. Proceedings of SoCTA 2017, Soft Computing: Theories and Applications, part of Advances in Intelligent Systems and Computing, Springer.

Mittal, A., Soundararajan, R. \& Bovik, A.C., 2012. Making a "completely blind" image quality analyzer. IEEE Signal Processing Letters, 20(3): 209-212.

Mohammadi, P., Ebrahimi-Moghadam, A. \& Shirani, S., 2014. Subjective and objective quality assessment of image: A survey. arXiv preprint arXiv:1406.7799.

Moorthy, A.K. \& Bovik, A.C., 2010. A two-step framework for constructing blind image quality indices. IEEE Signal processing letters, 17(5): 513-516.

Navas, K.A. \& Sasikumar, M., 2011. Image fidelity metrics: future directions. IETE Technical Review, 28(1): 50-56. 
Palus, H., 2006. Colorfulness of the image: definition, computation, and properties. Proceedings SPIE 6158, Lightmetry and Light and Optics in Biomedicine 2004, Warsaw, Poland.

Panetta, K., Gao, C. \& Agaian, S., 2013. No reference color image contrast and quality measures. IEEE transactions on Consumer Electronics, 59(3): 643-651.

Panetta, K.A., Wharton, E.J. \& Agaian, S.S., 2008. Human visual system-based image enhancement and logarithmic contrast measure. IEEE Transactions on Systems, Man, and Cybernetics, Part B (Cybernetics), 38(1): 174-188.

Panetta, K., Zhou, Y., Agaian, S. \& Jia, H., 2011. Nonlinear unsharp masking for mammogram enhancement. IEEE Transactions on Information Technology in Biomedicine, 15(6): 918-928.

Pech-Pacheco, J.L., Cristóbal, G., Chamorro-Martinez, J. \& Fernández-Valdivia, J., 2000. Diatom autofocusing in brightfield microscopy: a comparative study. Proceedings 15th International Conference on Pattern Recognition, ICPR-2000, Barcelona, Spain.

Peli, E., 1990. Contrast in complex images. Journal of the optical society of America A, 7(10): 2032-2040.

Ponomarenko, N., Jin, L., Ieremeiev, O., Lukin, V., Egiazarian, K., Astola, J., Vozel, B., Chehdi, K., Carli, M., Battisti, F. \& Kuo, C.-C.J., 2015. Image database TID2013: Peculiarities, results and perspectives. Signal Processing: Image Communication, 30: 5777.

Puth, M.-T., Neuhäuser, M. \& Ruxton, G.D., 2015. Effective use of Spearman's and Kendall's correlation coefficients for association between two measured traits. Animal Behaviour, 102: 77-84. 
Santos, A., Solorzano, C.O.D., Vaquero, J.J., Pena, J.M., Malpica, N. \& Pozo, F.D., 1997. Evaluation of autofocus functions in molecular cytogenetic analysis. Journal of microscopy, 188(3): 264-272.

Sharma, N. \& Verma, O.P., 2017. A novel fuzzy based satellite image enhancement. Proceedings of International Conference on Computer Vision and Image Processing (CVIP-2016), Roorkee, India.

Talebi, H. \& Milanfar, P., 2018. NIMA: Neural Image Assessment. IEEE Transactions on Image Processing, 27(8): 3998-4011.

Varga, D., 2021. Comprehensive evaluation of no-reference image quality assessment algorithms on authentic distortions. arXiv preprint arXiv:2011.07950v1

Video Quality Experts Group, 2003. Final report from the video quality experts group on the validation of objective models of video quality assessment, phase II. 2003 VQEG.

Vonikakis, V., Chrysostomou, D., Kouskouridas, R. \& Gasteratos, A., 2013. A biologically inspired scale-space for illumination invariant feature detection. Measurement Science and Technology, 24(7), 074024.

Wang, L., 2021. A Survey on Image Quality Assessment. arXiv preprint arXiv:2109.00347v1

Wharton, E., Agaian, S. \& Panetta, K., 2006. A logarithmic measure of image enhancement. Proceedings SPIE 6250, Mobile Multimedia/Image Processing for Military and Security Applications, Florida, United States.

Wu, S., Zhu, Q., Yang, Y. \& Xie, Y., 2013. Feature and contrast enhancement of mammographic image based on multiscale analysis and morphology. Proceedings of 2013 IEEE International Conference on Information and Automation (ICIA), Yinchuan, China.

Xie, Z.-X. \& Wang, Z.-F., 2010. Color image quality assessment based on image quality parameters perceived by human vision system. Proceedings of 2010 International Conference on Multimedia Technology, Ningbo, China. 
Xu, S., Jiang, S. \& Min, W., 2017. No-reference/blind image quality assessment: A survey. IETE Technical Review, 34(3): 223-245.

Yan, J., Li, J. \& Fu, X., 2019. No-reference quality assessment of contrast-distorted images using contrast enhancement. arXiv preprint arXiv:1904.08879.

Yendrikhovski, S.N., Blommaert, F.J.J. \& Ridder, H.D., 1998. Perceptually optimal color reproduction. Proceedings SPIE 3299, Human Vision and Electronic Imaging III, San Jose.

Zhai, G. \& Min, X., 2020. Perceptual image quality assessment: a survey. Science China Information Sciences, 63(11): 211301. 